'Laboratorio de Citogenética

Molecular, Instituto de Nutrición y Tecnología de los Alimentos (INTA), Universidad de Chile, Santiago, Chile. ${ }^{2}$ Manchester Centre for Genomic Medicine, Division of Evolution \& Genomic Sciences, School of Biological Sciences, Faculty of Biology, Medicine and Health, University of Manchester, Manchester, UK. aMédico, Genetista Clínico, $\mathrm{PhD}(\mathrm{c})$. bBioquímica, PhD. TTecnóloga Médica.

Fuente de financiamiento: Ninguno.

Recibido el 26 de abril de 2017, aceptado el 18 de julio de 2017.

Correspondencia a: Lorena Santa María Laboratorio de Citogenética Molecular, INTA, Universidad de Chile.

El Líbano 5524, PO 7830490, Santiago, Chile. Isantamaria@inta.uchile.cl

\section{Microarreglos cromosómicos en 236 pacientes chilenos con trastornos del neurodesarrollo y anomalías congénitas}

\author{
VÍCTOR FAUNDES ${ }^{1,2, a}$, LORENA SANTA MARÍA $^{1, b}$, PAULINA MORALES ${ }^{1, c}$, \\ BIANCA CUROTTO ${ }^{1, c}$, MARÍA ANGÉLICA ALLIENDE ${ }^{1, c}$

\section{Microarrays in 236 patients with congenital abnormalities} \\ neurodevelopmental disorders and
}

Background: In 20\% of neurodevelopmental disorders (NDD) and congenital abnormalities (CA) the cause would be a genomic imbalance detectable only by chromosomal microarrays (CMA). Aim: To analyze the results of CMA performed at the INTA Laboratory of Molecular Cytogenetics, during a period of four years in patients with NDD or CA. Material and Methods: Retrospective study that included all CMA reports of Chilean patients. Age, sex, clinical diagnosis and origin were analyzed, as well as the characteristics of the finding. The percentage of cases diagnosed by CMA was calculated considering all patients with pathogenic $(P V)$ or probably pathogenic variants $(V L P)$. Finally, we studied the association between patients' characteristics and a positive CMA outcome. Results: A total of 236 reports were analyzed. The median age was 5.41 (range 2.25-9.33) years, and 59\% were men. Ninety chromosomal imbalances were found, which corresponded mainly to deletions (53.3\%), with a median size of 1.662 (range 0.553-6.673) Megabases. The diagnostic rate of CMA in Chilean patients from all over the country was $19.2 \%$. There was a close relationship between the patient's sex and the detection of VLP/VP $(p=0.034)$. Conclusions: Our diagnostic rate and the association between female sex and a higher percentage of diagnosed cases are concordant with other international studies. Therefore, CMA is a valid diagnostic tool in the Chilean population.

(Rev Med Chile 2017; 145: 854-861)

Key words: Comparative Genomic Hybridization; Congenital Abnormalities; Neurodevelopmental Disorders.
L

os trastornos del neurodesarrollo (TN) son un grupo de condiciones que se inician y manifiestan en la infancia, usualmente antes de que el niño entre al colegio, y se caracterizan por déficits que alteran el funcionamiento personal, social, académico u ocupacional. Dentro de este grupo se incluyen trastornos tales como retraso de desarrollo psicomotor (RDSM), discapacidad intelectual (DI) o trastornos del espectro autista $(\mathrm{TEA})^{1}$. Por su parte, las anomalías congénitas
(AC) se definen como todas las alteraciones estructurales o funcionales que ocurren en el período intrauterino y se detectan durante el embarazo, en el parto o postnatalmente ${ }^{2}$. Aunque no existen cifras exactas en Chile, la prevalencia de DI sería $\sim 1,2 \%{ }^{3,4}$ y de TEA de $0,9 \%{ }^{5}$, mientras que las AC constituirían más de un tercio de las muertes infantiles en nuestro país ${ }^{6}$.

Las causas de estos trastornos y las AC son diversas, pero se sabe que cerca de $25 \%$ de los casos 
de DI y $30-40 \%$ de los casos de TEA tienen un origen genético ${ }^{7,8}$; similar porcentaje de casos con AC serían también de causa genética ${ }^{9}$. Establecer el diagnóstico etiológico en todos estos pacientes resulta fundamental, puesto que permite proveer un pronóstico, determinar riesgos de recurrencia, refinar los tratamientos y evitar procedimientos diagnósticos innecesarios, entre otras ventajas ${ }^{10}$. Por estos motivos y por la evidencia acumulada, los microarreglos cromosómicos (CMA, del inglés chromosomal microarrays) son el primer examen a realizar en todos los casos con RDSM, DI, TEA y AC sin una etiología evidente, pudiendo diagnosticar hasta 20\% de estos pacientes, quienes presentan alguna pérdida o ganancia de material cromosómico (CNV, del inglés copy-number variant) no detectable con el cariotipo convencional ${ }^{10,11}$.

En Chile, esta tecnología está disponible para uso clínico desde el año 2013 en nuestro laboratorio, la cual se ha incorporado posteriormente en otras dos instituciones. Existe un reporte previo chileno de Lay-Son y cols. ${ }^{12}$ que determinó que $25 \%$ de los pacientes con AC mayores, dismorfias faciales y/o RDSM/DI presentaban un desbalance cromosómico que explicaba su sintomatología. Si bien este estudio reflejó resultados algo similares a lo reportado internacionalmente ${ }^{11}$, se realizó con una cohorte pequeña $(n=40)$, de los cuales $12,5 \%$ ya tenía una alteración cromosómica no bien caracterizada por cariotipo convencional. Además, todos los pacientes provenían de un único hospital en el sur de Santiago.

El objetivo de este estudio fue analizar retrospectivamente los resultados de CMAs realizados en nuestro laboratorio en un período de 4 años para estimar la tasa de diagnóstico de estos $\mathrm{CNV}$ en pacientes con $\mathrm{TN}, \mathrm{AC} \mathrm{o}$ ambos, derivados de todo Chile.

\section{Materiales y Métodos}

\section{Reportes de pacientes}

Estudio retrospectivo que incluyó 236 reportes de CMA del total de 255 informados por nuestro laboratorio desde marzo de 2013 hasta enero de 2017. Se consignaron la edad, sexo, diagnóstico clínico y procedencia, así como las características del hallazgo. Con respecto al diagnóstico clínico, se clasificaron en 5 categorías, según el motivo principal de derivación: TN, AC, caracterización de cromosomopatía (CC), combinación de diagnós- ticos (CD) y sin diagnóstico (SD). Se excluyeron informes de pacientes provenientes del extranjero, mientras que el resto se categorizó en pacientes de la Región Metropolitana (RM) o de otras regiones. Además, se consideraron sólo los probandos, excluyéndose familiares de primer grado, para no distorsionar el cálculo final de la tasa diagnóstica. Finalmente, las características del hallazgo consideradas fueron número de variantes por paciente, tipo de CNV (deleción o amplificación), tamaño de la variante (en número de megabases), cromosoma afectado, número de genes registrados en la base de datos "Online Mendelian Inheritance in Man" (OMIM) causantes de enfermedad a la fecha del informe ${ }^{13}$, y el tipo de interpretación de la variante según las recomendaciones del Colegio Americano de Genética y Genómica Médica ${ }^{14}$ y europeas ${ }^{15}$. Brevemente, se consideran diversas características como el tamaño, número de genes afectados, presencia de la variante en población control, etc., para determinar su interpretación. Así, las variantes pueden interpretarse como patogénicas (VP), de significado incierto o benignas (VB); las variantes de significado incierto se subclasifican en probablemente patogénicas (VLP), probablemente benignas (VLB) y de significado incierto no subclasificable (VUS, del inglés variant of uncertain significance $)^{14,15}$.

\section{Métodos}

De cada paciente se recolectó $5 \mathrm{ml}$ de sangre en tubos con EDTA para la extracción de ADN usando el kit DNeasy ${ }^{\circledR}$ Blood\&Tissue y siguiendo el protocolo del fabricante (Qiagen Group, Alemania). Posteriormente, este ADN se procesó e hibridó en conjunto con ADN control usando la plataforma Agilent 8x60K, ISCA v2 (Agilent Technologies, Santa Clara, CA, USA); la máxima resolución del CMA en las regiones de mayor interés (regiones conocidas de microdeleciones, genes sensibles a dosis o causantes de TN/AC) va desde 25 kilobases $(\mathrm{Kb})$. Todos los procedimientos fueron llevados a cabo según las instrucciones del fabricante. Por último, el análisis se realizó usando el programa Agilent CytoGenomics y su algoritmo ADM-2, así como el navegador del genoma UCSC Genome Browser ${ }^{16}$.

\section{Análisis estadístico}

La edad, sexo, procedencia, categoría diagnóstica y las características de las variantes, según 
se detalló previamente, fueron descritos como frecuencias y porcentajes, o medianas y rangos intercuartiles (IQR), según corresponda. Se calculó el porcentaje de casos diagnosticados por CMA considerando todos los pacientes con VP o VLP del total de pacientes con cariotipo convencional normal incluidos en este estudio. Finalmente, comparamos la edad, sexo, procedencia y el tipo de diagnóstico clínico entre aquellos pacientes diagnosticados o no con alguna VP/VLP y cariotipo normal usando las pruebas de chi-cuadrado, $\mathrm{Z}$ para proporciones con corrección de Bonferroni, y Mann-Whitney para muestras no pareadas. Un valor $\mathrm{p}<0,05$ se consideró como estadísticamente significativo.

\section{Resultados}

De los 255 casos referidos a estudio por CMA a nuestro laboratorio durante el período descrito, 236 provenían de pacientes chilenos probandos $(92,5 \%)$, los cuales fueron seleccionados para el análisis. En la Tabla 1 y Figura 1 se describen las características generales de estos pacientes y las variantes en el número de copias halladas. Como se puede ver, la mediana de la edad fue de 5,41 $(2,25-9,33)$ años, con un leve predominio de hombres por sobre mujeres ( $58,9 \%$ versus $41,1 \%$, respectivamente). La mayoría de los casos provenían de la RM $(\mathrm{n}=191 ; 80,9 \%)$ y de las regiones X y V del país (7,2\% y 3,8\%, respectivamente). Por otra parte, los TN constituyeron el principal motivo de derivación $(\mathrm{n}=118 ; 50 \%)$, seguidos por $\mathrm{CD}(\mathrm{n}=48 ; 20,3 \%)$ y AC $(\mathrm{n}=14 ; 5,9 \%)$. Vale destacar que $18,6 \%$ de los pacientes fue derivado sin una hipótesis diagnóstica ni descripción clínica de las manifestaciones ( $\mathrm{n}=44$ ) (Figura 1A).

Con respecto a las características de las variantes del número de copias, se encontraron un total de $90 \mathrm{CNV}$, lo que equivale a 1 (1-1) CNV por paciente. Estas $\mathrm{CNV}$ correspondían principalmente a deleciones $(\mathrm{n}=48 ; 53,3 \%)$, tenían un tamaño de 1,662 (0,553-6,673) megabases y comprometían a 3 (1-8) genes OMIM causantes de enfermedad. Además, los principales cromosomas afectados fueron el $5(\mathrm{n}=10,11,1 \%), X(\mathrm{n}=10,11,1 \%)$, $15(\mathrm{n}=7,7,8 \%)$ y $7(\mathrm{n}=7,7,8 \%)$ (Figura $1 \mathrm{~B})$. Si se considera el número total de pacientes con cariotipo convencional normal $(n=224)$ y el tipo variantes que se encontró, resultó que en el 5,4\% y $13,8 \%$ de ellos se detectó al menos una VLP o VP, respectivamente (Figura 1C). Por lo tanto, la tasa de diagnóstico de los CMA en pacientes chilenos provenientes de todo el país fue de 19,2\% (Figura 1C).

Finalmente, al estudiar los principales factores asociados a un diagnóstico positivo por CMA en población chilena con cariotipo normal, hubo una estrecha relación entre el sexo del paciente y la detección de $\mathrm{CNV}$ patogénicas o posiblemente patogénicas $\left(\chi^{2}=4,48 ; \mathrm{df}=1 ; \mathrm{p}=0,034\right)$. De hecho, la proporción de mujeres diagnosticadas fue estadísticamente superior que aquellas que no lo fueron, e inversamente, la proporción de hombres diagnosticados fue estadísticamente inferior a la de

\section{Tabla 1. Características de los pacientes y los desbalances genéticos encontrados utilizando CMA}

\begin{tabular}{|ll|}
\hline Características & \\
\hline Pacientes Incluidos & $\mathrm{n}=236$ \\
- Edad (m [IQR]) & $5,41(2,25-9,33)$ \\
- Sexo (mujeres, $\mathrm{n}[\%])$ & $97(41,1)$ \\
- Procedencia (RM, $\mathrm{n}[\%])$ & $191(80,9)$ \\
Alteraciones o variantes genéticas detectadas & $\mathrm{n}=90$ \\
- Número de variantes por paciente (m [IQR]) & $1(1-1)$ \\
- Tipo de desbalance (deleciones, $\mathrm{n}[\%])$ & $48(53,3)$ \\
- Tamaño de la variante (en Mb, m [IQR])) & $1,662(0,553-6,673)$ \\
- Genes OMIM (m [IQR]) & $3(1-8)$ \\
\hline
\end{tabular}

Abreviaciones: $\mathrm{CMA}=$ microarreglo cromosómico; $\mathrm{IQR}=$ rango intercuartílico; $\mathrm{m}=$ mediana; $\mathrm{Mb}=$ megabases; OMIM = "Online Mendelian Inheritance in Man"; RM = Región Metropolitana. aSólo se consideraron los pacientes con variantes del número de copias $(n=71)$. b Genes OMIM causantes de enfermedad a la fecha de emisión del informe. 
A

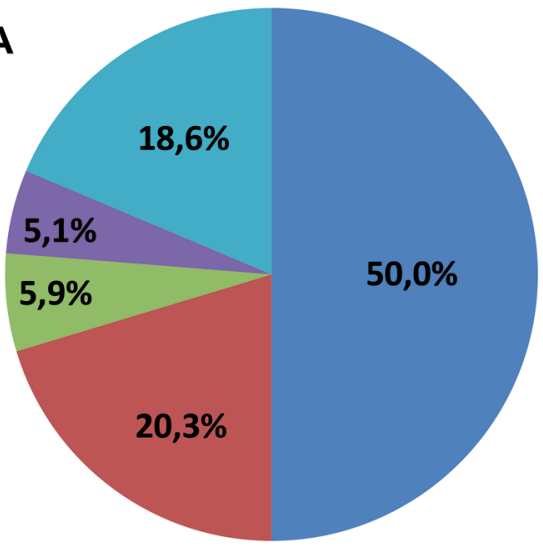

B

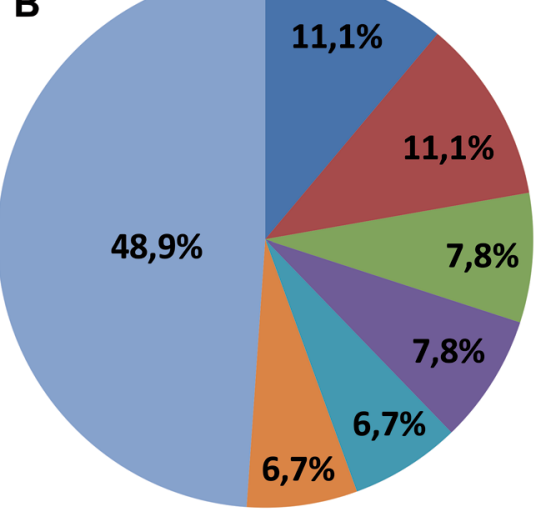

C
- Trastorno del Neurodesarrollo

Combinación de Diagnósticos

Anomalías Congénitas

Caracterización de Cromosomopatía

Sin Diagnóstico

Cromosoma X

Cromosoma 15

- Cromosoma 7

Cromosoma 8

Cromosoma 2

Otros cromosomas

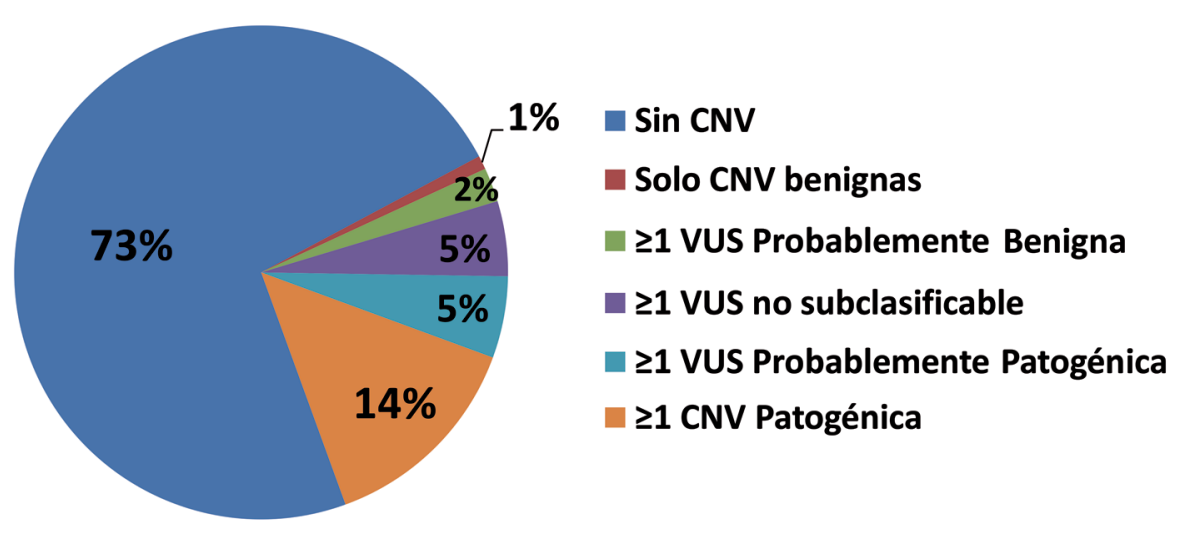

Figura 1. A) Diagnósticos de los pacientes referidos para estudio por microarreglo cromosómico $(n=236)$. B) Cromosomas comprometidos por deleciones/amplificaciones en los pacientes referidos para estudio por microarreglo cromosómico $(n=90)$. C) Interpretación de las variantes halladas en los pacientes con cariotipo normal referidos para estudio por microarreglo cromosómico $(\mathrm{n}=224)$. Abreviaciones: CNV = Copy-number variant o Variante del número de copias; VUS = Variant of uncertain significance o variante de significado incierto. 
Tabla 2. Asociación entre el diagnóstico por CMA en pacientes con cariotipo normal y sus diversas características

\begin{tabular}{|c|c|c|c|}
\hline Características & $\begin{array}{l}\text { Casos diagnosticados } \\
\qquad(n=43)^{a}\end{array}$ & $\begin{array}{l}\text { Casos no diagnosticados } \\
\qquad(n=181)^{a}\end{array}$ & Valor $p^{a}$ \\
\hline Edad (m [IQR]) & $5,25(1,5-9)$ & $5,74(2,7-9,5)$ & 0,57 \\
\hline Sexo (mujeres, n [\%]) & $24(55,8)$ & $69(38,1)$ & $0,034^{b}<0,05^{c}$ \\
\hline Procedencia (RM, n [\%]) & $36(83,7)$ & $145(80,1)$ & $0,589^{b}>0,05^{c}$ \\
\hline $\begin{array}{l}\text { Diagnósticos (n [\%]) } \\
\text { - Trastorno del neurodesarrollo } \\
\text { - Anomalías congénitas } \\
\text { - Combinación de diagnósticos } \\
\text { - Sin diagnóstico }\end{array}$ & $\begin{array}{r}17(39,5) \\
1 \quad(2,3) \\
13(30,2) \\
12(27,9)\end{array}$ & $\begin{array}{r}101(55,8) \\
13(7,2) \\
35(19,3) \\
32(17,7)\end{array}$ & $\begin{array}{l}0,077^{b} \\
>0,05^{c} \\
>0,05^{c} \\
>0,05^{c} \\
>0,05^{c}\end{array}$ \\
\hline
\end{tabular}

Abreviaciones: $\mathrm{CMA}=$ microarreglo cromosómico; $\mathrm{IQR}=$ rango intercuartil; $\mathrm{RM}=$ Región Metropolitana; $\mathrm{m}=\mathrm{mediana}$. ${ }^{\mathrm{a} E n}$ negrita las cifras estadísticamente significativas. ${ }^{b}$ Valor $\mathrm{p}$ para la prueba de $\chi^{2}$. ${ }^{`}$ Valor p para la prueba Z de proporciones con corrección de Bonferroni.

pacientes masculinos que no lo fueron ( $\mathrm{p}<0,05$ para ambas situaciones). También cabe destacar que existió una tendencia a detectarse un CMA alterado en aquellos pacientes con combinación de diagnósticos (CD) (Tabla 2).

\section{Discusión}

En el presente estudio se analizaron 236 resultados de CMA provenientes de igual número de probandos, derivados para estudio etiológico de RDSM, DI, TEA, AC y cromosomopatías. Se destaca en este trabajo el tamaño de la muestra, que sería la más grande reportada en Sudamérica ${ }^{12,17,18}$ y que se incluyeron pacientes provenientes de todo Chile, lo que contribuye, con mayor precisión, a conocer la prevalencia de este tipo de alteraciones genéticas en la población chilena. Por otro lado, es importante destacar que la asociación de un diagnóstico positivo por CMA con factores como el sexo del paciente, edad y el tipo de diagnóstico no habían sido descritos antes en población chilena.

$\mathrm{Al}$ comparar nuestros resultados con aquellos reportados por Lay-Son y cols. ${ }^{12}$, ambos estudios muestran una mayor derivación de pacientes del sexo masculino por sobre el femenino. Esto se explicaría por la mayor prevalencia de TN en hombres que en mujeres, reflejando la contribución de enfermedades ligadas al cromosoma X recesivas en dicha diferencia ${ }^{19} \mathrm{y}$ un "factor protector femenino" frente a este tipo de trastornos ${ }^{20}$. En relación a la edad de los probandos estudiados, la mediana de la edad se encuentra alrededor de los 5 años en ambos estudios. Tanto los TN como las AC se manifiestan en la infancia, lo que amerita su derivación en esta etapa de la vida ${ }^{1,2}$.

La tasa de diagnóstico de 19\% estimada en el presente estudio podría ser considerada como la prevalencia de estos desbalances en población chilena con TN, AC o ambos, lo que concuerda además con las cifras promedio reportadas internacionalmente (entre 15 a $20 \%)^{11,17,18}$. Sin embargo, esta podría ser mayor, ya que en el estudio previo realizado en Chile ${ }^{12}$ se detectó variantes clínicamente significativas en $25 \%$ de los pacientes, porcentaje que sube a cerca de $29 \%$, si se consideran solo aquellos pacientes con cariotipo convencional normal. Otros parámetros, como el número de desbalances detectados, cromosomas comprometidos y tamaño de los desbalances, no se pueden comparar entre ambos estudios ${ }^{12}$, ya que solo 9 pacientes presentaron variantes clínicamente significativas detectadas por CMA y cariotipo convencional normal en el estudio previo ${ }^{12}$.

$\mathrm{Al}$ realizar el análisis asociativo entre un resultado alterado por CMA y diversas características de los pacientes con cariotipo normal, detectamos una clara asociación entre el sexo del paciente y la posibilidad de encontrar una VLP/VP. Esta situación confirma para la población chilena lo descrito en estudios internacionales ${ }^{20,21}$. Se sabe que las mujeres presentarían un "factor protector" para el desarrollo de TN. Por lo tanto, para una paciente femenina diagnosticada con alguno 
de estos trastornos, la probabilidad de encontrar una etiología genética es mayor, ya sea por algún desbalance cromosómico ${ }^{20}$ o por mutaciones puntuales deletéreas ${ }^{21}$. Aunque la explicación exacta para este fenómeno no se conoce, se ha visto que las variantes genéticas que regularían rasgos antropométricos y polimorfismos en el cromosoma X darían cuenta de esta diferencia ${ }^{22}$. Por otra parte, aunque no encontramos una clara asociación, existe una tendencia a detectarse un mayor porcentaje de casos con CMA positivo si fueron derivados con CD. Esto también ha sido descrito en estudios previos en otras poblaciones $^{23}$, lo que se explica por la pleiotropía de la mayoría de las alteraciones cromosómicas al presentar múltiples manifestaciones en diversos sistemas y órganos ${ }^{24}$. Además de estas asociaciones, es importante destacar que 27,9\% de los casos derivados sin diagnósticos ni información clínica pudieron ser finalmente clarificados con CMA. Esta es una situación excepcional, ya que se sabe que la información clínica también resulta relevante para una correcta interpretación de los hallazgos ${ }^{25}$, por lo que resulta imperioso que los clínicos familiarizados con este tipo de exámenes deriven a sus pacientes con la mayor información posible. Nuestro laboratorio dispone de una ficha de fenotipo que facilita este proceso y que se puede descargar desde la página web (véase https://www. inta.cl/sites/default/files/ficha_fenotipo_acgh_0. pdf), con la cual sólo deben marcar aquellas manifestaciones presentes en el/la paciente. Por último, no se compararon las características de las variantes halladas por CMA entre aquellos pacientes con diagnóstico, ya que el tamaño y el número de genes comprometidos son criterios para definir si una variante es o no patogénica, según las recomendaciones internacionales ${ }^{14,15}$. Por ende, las diferencias que se pudieran encontrar se deben a la clasificación misma.

Otro punto a destacar de este trabajo es que se confirman los resultados obtenidos en un reporte previo sobre rearreglos subteloméricos ${ }^{26}$. En ese análisis previo, determinamos que $8,9 \%$ de los pacientes chilenos con TN, AC o ambos presenta un desbalance en las regiones subteloméricas, comprometiendo principalmente los cromosomas $2,5,8$ y 15 , entre otros. Si lo comparamos con las cifras determinadas por CMA, 20 pacientes del total de la muestra $(\mathrm{n}=236)$ presentó al menos un desbalance subtelomérico, comprometiendo los mismos cromosomas descritos en el estudio anterior, lo que equivale a $8,5 \%{ }^{26}$. Además, $17 / 224$ pacientes con cariotipo normal tenía este tipo de desbalances. Cabe destacar que ambas muestras son independientes, por lo que se corrobora que en $\sim 8 \%$ de los pacientes chilenos con TN, AC o ambos, la causa de dichos trastornos son CNV que involucran las regiones subteloméricas, y que la utilización del CMA aumentan dicha tasa diagnóstico en $11 \%$, al detectar microdeleciones/ duplicaciones intersticiales.

Si bien el presente estudio no consideró una muestra representativa, ya que no es prospectivo, y el CMA es un examen costoso (alrededor de US\$ 1.000 por paciente) y no codificado por FONASA, es importante señalar que un porcentaje importante $(29 \%)$ de los pacientes fueron derivados desde hospitales públicos y como parte del estudio etiológico de RDSM, DI o TEA, como recomiendan las guías internacionales al respecto ${ }^{7,10}$. Considerando lo anterior y las tasas de diagnóstico estimadas de los CMA en población chilena con TN/AC (19-29\%), es urgente que FONASA lo incorpore dentro de las prestaciones codificadas o se dispongan de recursos para su realización y así ampliar el acceso al estudio etiológico de TN y AC para una oportuna intervención terapéutica, entregar un pronóstico y determinar riesgos de recurrencia a las familias afectadas.

\section{Lista de abreviaciones}

AC: Anomalías congénitas.

CC: Caracterización de cromosomopatía.

CD: Combinación de diagnósticos.

CNV: Variante del número de copias.

DI: Discapacidad intelectual.

IQR: Rango intercuartil.

$\mathrm{Kb}$ : kilobases.

OMIM: "Online Mendelian Inheritance in Man". RDSM: Retraso del desarrollo psicomotor.

RM: Región Metropolitana.

SD: Sin diagnóstico.

TEA: Trastornos del espectro autista.

TN: Trastornos del neurodesarrollo.

VB: Variante benigna.

VLB: Variante probablemente benignas.

VLP: Variante probablemente patogénica.

VP: Variante patogénica.

VUS: Variante de significado incierto no subclasificable. 


\section{Referencias}

1. American Psychiatric Association. Neurodevelopmental Disorders. En: American Psychiatric Association, editor, Diagnostic and statistical manual of mental disorders. Arlington, VA: American Psychiatric Publishing; 2013.

2. Organización Mundial de la Salud. Anomalías congénitas. OMS; 2015 [actualizado en abril de 2015; citado 7 de febrero de 2017]; Disponible en: http://www.who.int/ mediacentre/factsheets/fs370/es/.

3. Fondo Nacional de la Discapacidad. Primer estudio nacional de la discapacidad en Chile. Santiago, Chile: Ograma S.A.; 2005. Disponible en: http://www.senadis. cl/pag/136/1196/resultados_endisc_i.

4. Servicio Nacional de la Discapacidad. Segundo estudio nacional de la discapacidad en Chile. Santiago, Chile: Feyser Ltda.; 2016. Disponible en: http://www.senadis. cl/pag/355/1197/ii_estudio_nacional_de_discapacidad.

5. MINISTERIO DE SALUD. Guía de Práctica Clínica de Detección y Diagnóstico Oportuno de los Trastornos del Espectro Autista (TEA). Santiago, Chile: MINSAL; 2011. Disponible en: http://web.minsal.cl/portal/url/item/ bd81e3a09ab6c3cee040010164012ac2.pdf.

6. Dipierri JE, Acevedo NE, Bronberg RA. Mortalidad infantil por malformaciones congenitas en Chile: analisis temporal y espacial, 1997-2011. Rev Panam Salud Publica 2015; 38 (5): 380-7.

7. Schaefer GB, Mendelsohn NJ, Professional Practice and Guidelines Committee. Clinical genetics evaluation in identifying the etiology of autism spectrum disorders: 2013 guideline revisions. Genet Med 2013; 15 (5): 399407.

8. Srivastava AK, Schwartz CE. Intellectual disability and autism spectrum disorders: causal genes and molecular mechanisms. Neurosci Biobehav Rev 2014; 46 Pt 2: 16174.

9. Rojas M, Walker L. Congenital malformations: general and genetic aspects. Int J Morphol 2012; 30 (4): 1256-65.

10. Moeschler JB, Shevell M, Committee on Genetics. Comprehensive evaluation of the child with intellectual disability or global developmental delays. Pediatrics 2014; 134 (3): e903-18.

11. Miller DT, Adam MP, Aradhya S, Biesecker LG, Brothman AR, Carter NP, et al. Consensus statement: chromosomal microarray is a first-tier clinical diagnostic test for individuals with developmental disabilities or congenital anomalies. Am J Hum Genet 2010; 86 (5): 749-64.

12. Lay-Son G, Espinoza K, Vial C, Rivera JC, Guzmán ML, Repetto GM. Chromosomal microarrays testing in children with developmental disabilities and congenital anomalies. J Pediatr (Rio J) 2015; 91 (2): 189-95.

13. Amberger JS, Bocchini CA, Schiettecatte F, Scott AF, Hamosh A. OMIM.org: Online Mendelian Inheritance in Man ( $\mathrm{OMIM}(\mathrm{R}))$, an online catalog of human genes and genetic disorders. Nucleic Acids Res 2015; 43 (Database issue): D789-98.

14. Kearney HM, Thorland EC, Brown KK, Quintero-Rivera F, South ST, Working Group of the American College of Medical Genetics Laboratory Quality Assurance Committee. American College of Medical Genetics standards and guidelines for interpretation and reporting of postnatal constitutional copy number variants. Genet Med 2011; 13 (7): 680-5.

15. Vermeesch JR, Brady PD, Sanlaville D, Kok K, Hastings RJ. Genome-wide arrays: quality criteria and platforms to be used in routine diagnostics. Hum Mut 2012; 33 (6): 906-15.

16. Rosenbloom KR, Armstrong J, Barber GP, Casper J, Clawson H, Diekhans M, et al. The UCSC Genome Browser database: 2015 update. Nucleic Acids Res 2015; 43 (Database issue): D670-81.

17. Krepischi-Santos AC, Vianna-Morgante AM, Jehee FS, Passos-Bueno MR, Knijnenburg J, Szuhai K, et al. Whole-genome array-CGH screening in undiagnosed syndromic patients: old syndromes revisited and new alterations. Cytogenet Genome Res 2006; 115 (3-4): 254-61.

18. Vianna GS, Medeiros PF, Alves AF, Silva TO, Jehee FS. Array-CGH analysis in patients with intellectual disability and/or congenital malformations in Brazil. Genet Mol Res. 2016; 15 (1): gmr.15017769.

19. Gecz J, Shoubridge C, Corbett M. The genetic landscape of intellectual disability arising from chromosome X. Trends Genet 2009; 25 (7): 308-16.

20. Jacquemont S, Coe BP, Hersch M, Duyzend MH, Krumm N, Bergmann S, et al. A higher mutational burden in females supports a "female protective model" in neurodevelopmental disorders. Am J Hum Genet 2014; 94 (3): 415-25.

21. Deciphering Developmental Disorders Study. Prevalence and architecture of de novo mutations in developmental disorders. Nature 2017; 542 (7642): 433-8.

22. Mitra I, Tsang K, Ladd-Acosta C, Croen LA, Aldinger KA, Hendren RL, et al. Pleiotropic Mechanisms Indicated for Sex Differences in Autism. PLoS Genet 2016; 12 (11): e1006425.

23. Syrmou A, Tzetis M, Fryssira H, Kosma K, Oikonomakis V, Giannikou K, et al. Array comparative genomic hybridization as a clinical diagnostic tool in syndromic and nonsyndromic congenital heart disease. Pediatr Res 2013; 73 (6): 772-6. 
24. Plona K, Kim T, Halloran K, Wynshaw-Boris A. Chromosome therapy: Potential strategies for the correction of severe chromosome aberrations. Am J Med Genet C Semin Med Genet 2016; 172 (4): 422-30.

25. Riggs ER, Jackson L, Miller DT, Van Vooren S. Phenotypic information in genomic variant databases enhances clinical care and research: the International
Standards for Cytogenomic Arrays Consortium experience. Hum Mut 2012; 33 (5): 787-96.

26. Santa María L, Faundes V, Curotto B, Morales P, Morales $\mathrm{K}$, Aliaga $\mathrm{S}$, et al. Comparison of two subtelomeric assays for the screening of chromosomal rearrangements: analysis of 383 patients, literature review and further recommendations. J App Genet 2016; 57 (1): 63-9. 